\section{A Rare Case of Peripheral Osteoma of the Zygoma}

Dae Young Kim, Kap Sung Oh

Department of Plastic Surgery, Samsung Medical Center, Sungkyunkwan University School of Medicine, Seoul, Korea

Correspondence: Kap-Sung Oh

Department of Plastic Surgery, Samsung Medical Center, Sungkyunkwan University School of Medicine, 81 Irwon-ro, Gangnam-gu, Seoul 135-710, Korea

Tel: +82-2-3410-2235, Fax: +82-2-3410-0036

E-mail: kapsung.oh@samsung.com

No potential conflict of interest relevant to this article was reported.

Received: 5 May 2014 • Revised: 30 May 2014 • Accepted: 30 May 2014 pISSN: 2234-6163 • elSSN: 2234-6171

http://dx.doi.org/10.5999/aps.2015.42.1.103 • Arch Plast Surg 2015;42:103-105

Copyright (C) 2015 The Korean Society of Plastic and Reconstructive Surgeons This is an Open Access article distributed under the terms of the Creative Commons Attribution Non-Commercial License (http://creativecommons.org/licenses/by-nc/3.0/) which permits unrestricted non-commercial use, distribution, and reproduction in any medium, provided the original work is properly cited.

Osteomas are a benign, slow growing osteogenic tumors consisting of well-differentiated mature bone tissue [1]. Osteomas are classified as peripheral, central, or extraskeletal and peripheral is the most common of the three. Generally, the mandible and paranasal sinuses are the most common locations of peripheral osteomas in the craniofacial region [2]. However, osteoma of the zygoma is rare. We report a

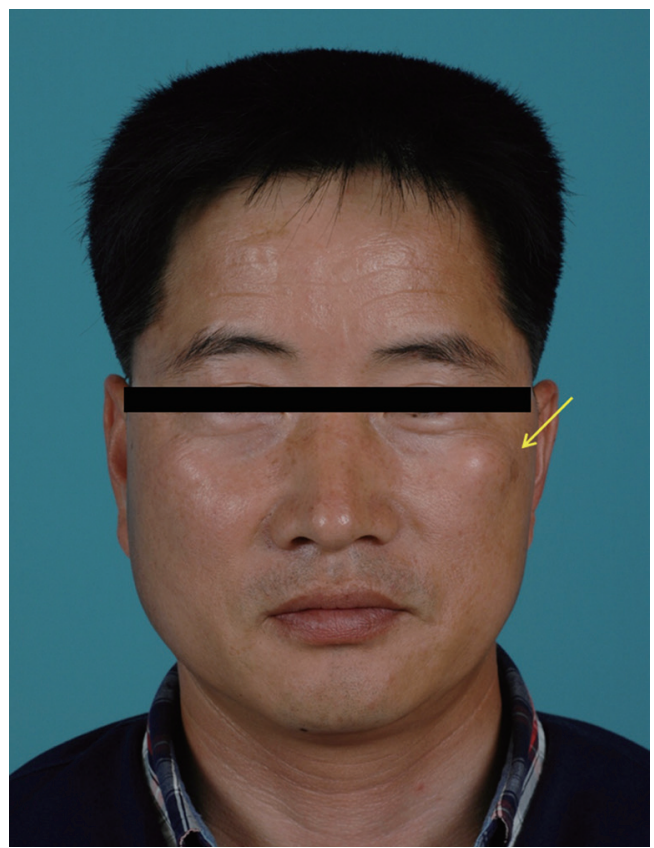

Fig. 1.

Preoperative photograph. A 41-year-old male with a firm mass on the left malar area (yellow arrow). rare case of peripheral osteoma of the zygoma. A 41-year-old male who was referred to our hospital presented with a painless firm mass in the left malar area in January 2013. The patient first noticed this lesion 10 years earlier and became concerned as it slowly enlarged. There was no history of a specific medical event, such as trauma. The patient also did not have systemic health problems. On examination, a well-circumscribed, round and firm mass was observed on the upper border of the left zygoma. The mass was about $1.5 \times 1.5 \mathrm{~cm}$. There were no signs or symptoms other than swelling (Fig. 1). A computed tomography (CT) scan revealed a mass protruding from the outer cortex of the left zygoma to adjacent the anterior cheek. The exophytic mass arose from the anterior wall of the left zygoma. It appeared lobulated (Fig. 2). On the basis of the clinical and radiographic findings, a diagnosis of osteoma was made. Under general anesthesia, the bony mass was excised through a transoral approach. The gross surgical specimen was a lobulated hard bony mass (Fig. 3). It measured about $1.2 \times 1.0 \mathrm{~cm}$. On histopathological examination, the specimen appeared to contain normal mature compact bone (Fig. 4). The diagnosis of osteoma was confirmed. One day after surgery, the patient was discharged without any complications. There was no clinical or radiographic evidence of recurrence on follow-up visit 1 month after the surgery (Fig. 5). Osteomas are benign osteogenic lesions that grow slowly. They occur due to proliferation of either compact or cancellous bone $[2,3]$. Based on the location, there are three variants. Peripheral (periosteal) osteomas arise from the periosteum and proliferate as sessile or

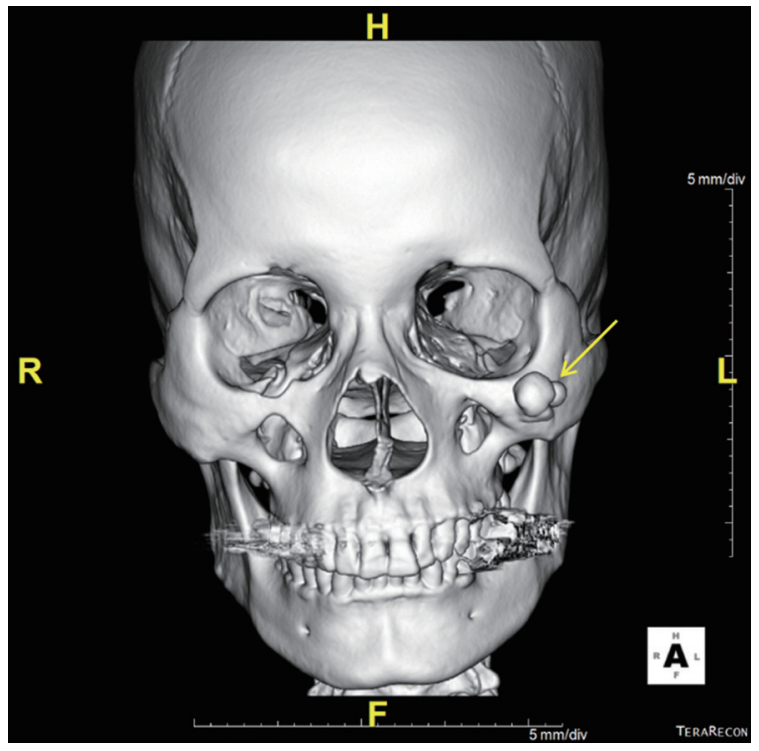

Fig. 2.

Preoperative computed tomography (CT). CT scan shows a lobulated bony mass (yellow arrow) protruding from the outer cortex of the left zygoma. 
Fig. 3.

Intraoperative photograph. Macroscopic specimen showing the lobulated bony mass.
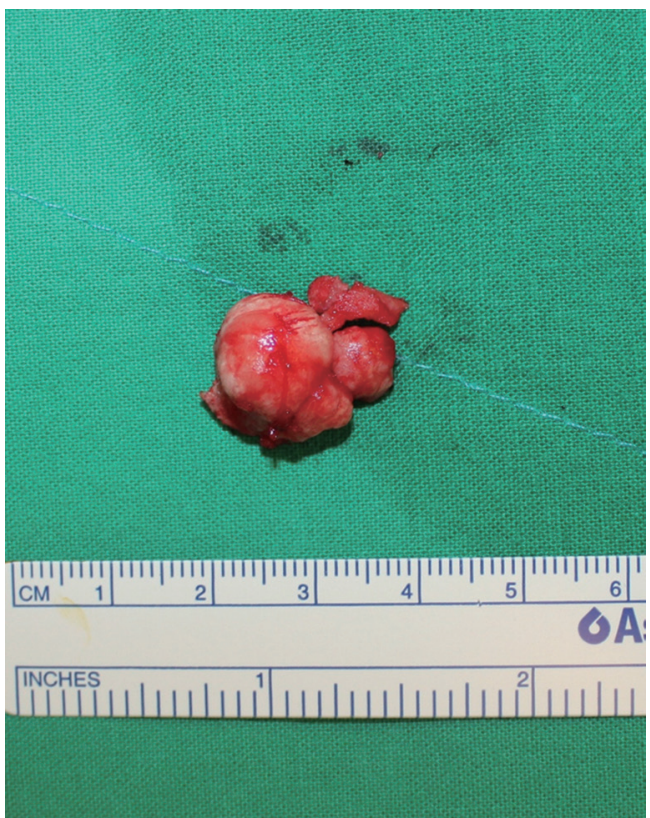

polypoid tumors. They are the most common variant of osteomas. Central (endosteal) osteoma develop as tumors on the endosteum. Extraskeletal osteomas (osseous choristoma) arise in soft tissue. Case reports of extraskeletal osteomas are rare $[1,4]$. Though various hypotheses have been suggested, the pathogenesis of osteomas has not been completely explained. Nevertheless 3 theories of the pathogenesis of osteomas have been accepted: developmental, traumatic, and infectious. The developmental theory suggests that osteomas arise either from embryonic cartilaginous remnants or from a persistent embryological periosteum $[2,4]$. Kaplan et al. [5] suggested that osteomas may be reactive lesions caused by trauma or muscle traction. The inflammatory theory suggests that chronic inflammation of the paranasal sinuses stimulates proliferation of periosteum-related osteogenetic cells $[2,4]$. The most common locations of osteomas in the craniofacial region are the paranasal sinuses and jawbones. Osteomas may also be found in orbital wall, pterygoid process, temporal bone, external ear canal, but osteomas of the zygoma are unusual [2]. Osteomas can occur at any age but are more frequent in young adults. They are more common in males than in females [2]. Our patient was a 41-year-old male. Generally, osteomas present as slow-growing and asymptomatic features, so early-stage osteomas are diagnosed incidentally on radiological examinations. Some fast growing osteomas or latestage, large osteomas can have mass-related

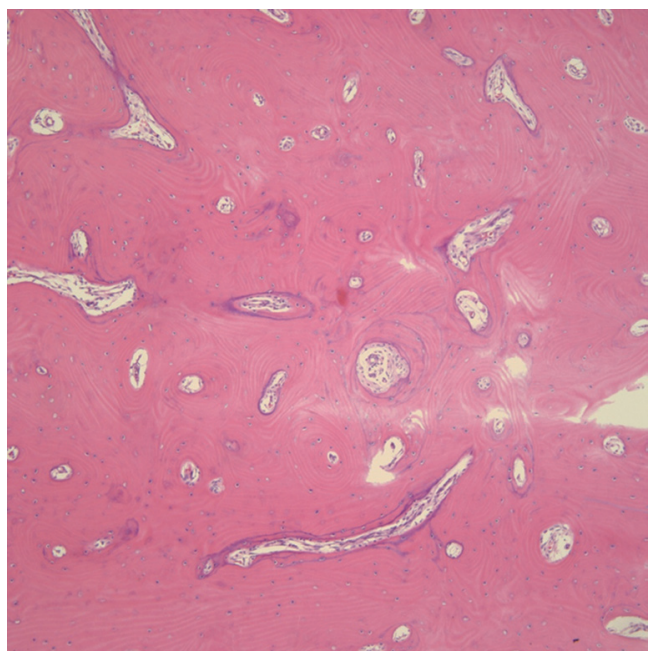

Fig. 4.

Histopathologic findings of the osteoma. The lesion consists of dense and lamellar cortical bone ( $H \& E_{\text {, }}$ $\times 100)$.

symptoms, which can deform the bone and compress adjacent structures [2]. Usually a conventional radiograph is sufficient to make a diagnosis, but CT is needed in some cases. Osteomas appear as oval, radiopaque, well-circumscribed masses attached by a broad base or pedicle to the host bone cortex in conventional radiographs. They appear as a smoothly demarcated, lobulated, homogenously hyperdense masses in CT [2]. Histologically, there are 2 osteoma variants. Compact osteomas comprise dense, compact bone with few marrow spaces and only a few osteons. Cancellous osteomas are characterized by bony trabeculae and fibrofatty marrow enclosing osteoblasts and with an architecture resembling mature bone [1]. The differential diagnosis includes hamartoma, peripheral ossufying fibroma, osteoid osteoma, periosteal osteoblastoma, and paraosteal osteosarcoma [1]. Most hamartomas stop growing after puberty. Osteomas are not directly related to development. Peripheral ossifying fibromas are reactive focal tumors. On radiological examination, peripheral ossifying fibromas present as radiopaque masses, like osteomas, but tend not to intrude into the osseous cortex. Contrary to osteomas, osteoid osteomas present as painful and rapid growing tumors. Periosteal osteoblastomas also present as painful and rapid growing features. Periosteal osteoblastomas are more round or ovoid than pedunculated. Paraosteal osteosarcomas are lobulated and sclerotic tumors. They grow fast, and patients with paraosteal osteosarcomas present elevated serum 
alkaline phosphatase $[1,4]$. Treatments for ostemas are controversial; however most agree that asymptomatic osteomas may not require surgical intervention while symptomatic osteomas definitely require surgical intervention. Rapidly growing osteomas can become symptomatic with time, so they should also be removed [4]. Peripheral osteomas usually appear as a pedunculated mushroom-like mass, so surgical excision is usually simple [2]. The surgical approach depends on the location, extent, and existing. We used an intraoral approach to remove an osteoma of the zygoma. Recurrence after surgical excision of ostema is rare. There are no reports of malignant transformation of an osteoma in the English literature [1]. This rare osteoma of the zygoma was seen in a 41-year-old male with a firm mass on left cheek that had been for 10 years. The mass was painless, slow growing, well-circumscribed, and round. On a CT scan, the mass was $1.2 \times 1.0 \mathrm{~cm}$ and lobulated like an osteoma. The mass was surgically removed. Under histopathological examination the specimen appeared as normal cortical bone. A diagnosis of osteoma was made. The patient was asymptomatic with no evidence of recurrence at 1 month after surgery.

\section{References}

1. Johann AC, de Freitas JB, de Aguiar MC, et al. Peripheral osteoma of the mandible: case report and review of the literature. J Craniomaxillofac Surg 2005;33:276-81.

2. Larrea-Oyarbide N, Valmaseda-Castellon E, Berini-

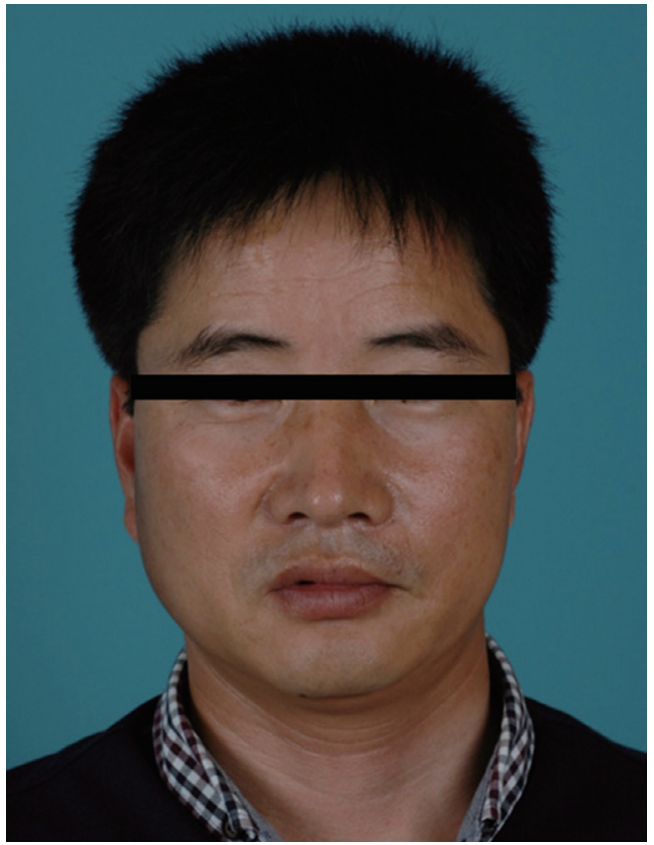

Fig. 5.

Postoperative 1-month images. There is no evidence of recurrence.
Aytes L, et al. Osteomas of the craniofacial region. Review of 106 cases. J Oral Pathol Med 2008;37:38-42.

3. Furlaneto EC, Rocha JR, Heitz C. Osteoma of the zygomatic arch: report of a case. Int J Oral Maxillofac Surg 2004;33:310-1.

4. Shanavas M, Chatra L, Shenai P, et al. Multiple peripheral osteomas of forehead: report of a rare case. Ann Med Health Sci Res 2013;3:105-7.

5. Kaplan I, Calderon S, Buchner A. Peripheral osteoma of the mandible: a study of 10 new cases and analysis of the literature. J Oral Maxillofac Surg 1994;52:467-70. 